\title{
Introducción \\ Salud Mental e Infancia: Una tarea pendiente
}

\author{
Introduction \\ Mental Health and Childhood: An Unfinished Task
}

\author{
Paola Ilabaca Baeza ${ }^{1}$ \\ Centro Cielo, Universidad Santo Tomás, Santiago, Chile
}

La niñez constituye una etapa crucial en el desarrollo humano y, en este sentido, las experiencias personales influyen en su pleno desarrollo. Por este motivo, la interacción del niño, en tanto entidad biológica, con su medio ambiente y con el sistema social, constituye uno de los pilares fundamentales para un desarrollo humano saludable (Brofenbrenner, 1979).

En este contexto, el reconocimiento del papel de la salud mental en la infancia constituye un eje primordial para la mayoría de los países del mundo, destacándose su relevancia en la mayoría de los programas de salud mundial. Es así como la Organización Mundial de la Salud (OMS) en octubre de 2013 celebró el día mundial de la salud mental, con una orientación especial dirigida a la salud mental de los/as niños/as y adolescentes. Un objetivo fundamental de este día fue concentrar la atención en la identificación, tratamiento y prevención de los trastornos emocionales y de conductas que presentan los menores de 18 años.

Para la OMS existen tres áreas fundamentales respecto a la salud mental infantil, la primera de ellas corresponde a los trastornos depresivos que cursan tanto en la infancia como en la adolescencia, y que se encuentran asociados a un importante desajuste social, relacionándose de manera directa con la segunda área prioritaria destacada por la OMS, a saber, el riesgo suicida, siendo este la tercera causa de muerte de adolescentes en el mundo. Por último, el tercer aspecto a destacar por la OMS corresponde a los trastornos conductuales, cuyo inicio se da en la infancia y tiende a persistir en la edad adulta de diferentes formas: abuso de drogas, delincuencia juvenil, conducta antisocial, problemas de relación con el otro (interpersonales y maritales), desempleo y una deteriorada salud física (OMS, 2003).
Por este motivo, la solución de los problemas de salud mental hoy en día constituye, de manera indiscutible, una tarea relevante tanto para los profesionales de la salud como para toda la sociedad en general, puesto que el impacto de una salud mental deteriorada no solo tiene un efecto negativo en la persona afectada, sino que también el costo se traspasa a todo su entorno, esto es, desde el más próximo (la familia) hasta el más macro (la sociedad), y no solo en términos psicológicos y emocionales, sino también con un alto costo económico.

En el contexto chileno, la importancia respecto a la salud mental, en general, comienza a relevarse en la última década del siglo XX (Abarzúa \& González, 2007). Así, a principio del año 2000, el Ministerio de Salud [MINSAL] desarrolla el Plan Nacional de Salud Mental y Psiquiatría (MINSAL, 2000). El plan tiene como objetivo contribuir a que tanto las personas como sus familias y comunidades logren la mayor capacidad posible para relacionarse entre sí y con el medio ambiente, de manera que se promueva el bienestar subjetivo, el desarrollo y uso de las potencialidades tanto psicológicas, cognitivas, afectivas como relacionales; además, por supuesto, del logro de las metas individuales y colectivas acorde con la justicia y el bien común (MINSAL, 2000). En este plan las prioridades definidas fueron:

Promoción y prevención de la salud mental.

Trastornos de la salud mental asociados a la violencia: maltrato infantil, violencia intrafamiliar y represión política (1973-1990).

Depresión

Trastornos de hiperactividad y atención en niños y adolescentes escolares.

Trastornos psiquiátricos severos.

Alzheimer y otras demencias.

Abuso y dependencia de alcohol y/o drogas.

\footnotetext{
1 Psicóloga, Universidad Santo Tomás. Doctora en Psicología, Universidad de Salamanca. Investigadora Centro CIELO, Universidad Santo Tomás. E-mail: pilabaca@santotomas.cl.
} 
Y en este sentido, la figura de la salud mental infantil y adolescente cobra relevancia, particularmente en lo relacionado con los trastornos de hiperactividad y de la atención, y más aún en aquellos asociados a la violencia, a los trastornos psiquiátricos severos y al abuso y dependencia del alcohol y/o drogas. Sin embargo, en un análisis realizado por Minoletti y Zaccaria (2005), respecto al Plan Nacional de Salud Mental en Chile, se da cuenta de una serie de falencias y dificultades encontradas, entre las que destaca aquella que hace alusión a la salud mental infantil. Estos autores mencionan que, a pesar de ser una de las prioridades programáticas del Plan Nacional de Salud Mental y Psiquiatría y uno de los objetivos sanitarios del MINSAL, el desarrollo de servicios de salud mental infantil está muy lejos de alcanzar la cobertura mínima definida inicialmente en el propio Plan, debido, según los autores, a que las autoridades no han comprometido efectivamente los recursos solicitados inicialmente para esta área, un hecho que finalmente se traduce en una amenaza para la consolidación de esta área.

En esta misma línea, la OMS realizó su propia evaluación al sistema de salud mental chileno (OMS, 2006). De ella, se destacan las siguientes deficiencias: a) la existencia de un $30 \%$ de centros ambulatorios de salud mental que no atienen a niños/as ni adolescentes; b) Un bajo porcentaje ( $28 \%$ ) de niños/as y adolescentes atendidos en centros ambulatorios y d) la existencia de solo 2 hospitales de día para adolescentes y 1 de servicio de corta estadía para niños/as y adolescentes en todo el país. Otro aspecto que salió a la luz en el informe de la OMS fue el escaso número de escuelas (7\%) que cuentan con horas de profesionales de la psicología, y de las que cuentan, menos del $20 \%$ realizan acciones de promoción de la salud mental.

Por este motivo, creemos que el abordaje temprano a problemáticas en la salud mental en la población infanto-juvenil, constituye un eje fundamental para prevenir $\mathrm{y} / \mathrm{o}$ reducir futuras patologías mentales a largo plazo, puesto que invertir en la salud mental de nuestros niños hoy nos asegura que nuestros adultos del futuro cuenten con mejores herramientas para enfrentar el día a día.

En este sentido, la presente sección especial de la Revista Summa Psicológica UST está orientada a reflexionar en torno a la salud mental e infancia, por este motivo, hemos recogido 5 investigaciones que dan cuenta de diferentes aspectos sobre infancia y salud mental. En el primero de ellos, Raúl Morales Villegas nos presenta la relación que existe entre el nivel socioeconómico, habilidades intelectuales y desnutrición en dos de las poblaciones indígenas más vulnerables de México, los Tenek y Nahúa. Para ello, se seleccionaron dos comunidades: Cuatlamayan y Tocoy, donde viven indígenas de las etnias antes citada. Los autores destacan que el déficit en el desarrollo cognitivo de los niños indígenas, que viven en situación de pobreza, debe ser un llamado a las autoridades para que se diseñen e implementen políticas públicas específicas y focalizadas en las condiciones sociales, económicas y de salud de este grupo de niños y sus pueblos o comunidades de referencia.

Por otro lado, Gabriel Abarca Brown analiza en su artículo el estado actual de las publicaciones en revistas de psicología a nivel nacional respecto del tema de la salud mental en población infanto-juvenil inmigrante. Se destaca en este artículo los escasos estudios realizados en Chile sobre salud mental en grupos minoritarios, en este caso, en población inmigrante, y más precisamente, sobre las de barreras de diferentes tipos que impide a este colectivo acceder a una atención sanitaria de calidad.

Por otra parte, Francisca Pérez nos introduce en los beneficios de una paternidad activa en el desarrollo integral de niños y niñas. Este artículo busca introducir una herramienta que amplíe la mirada del desarrollo infantil y las relaciones al interior de la familia, considerando la interacción madre-padre-hijo/a.

En el ámbito de la violencia, Hugo Plaza Villarroel indaga sobre la relación entre el procesamiento traumatogénico de experiencias de violencia sexual en niñas, con características de la victimización sufrida. Los autores destacan que la investigación realizada constituye una aproximación empírica frente a percepciones y atribuciones asociadas a la victimización sexual infantil en Chile y que podría contribuir al desarrollo de otras investigaciones que profundicen sobre el efecto mediador que tienen las atribuciones y percepciones que hace la víctima sobre el evento traumático y su posterior ajuste psicológico.

Finalmente, y desde la superación de estas problemáticas, Cristian Pinto Cortez expone un análisis sobre el concepto de resiliencia y sus diferentes etapas de investigación a través del tiempo, realizando una integración de este modelo positivo en la comprensión y abordaje de la victimización infanto-juvenil.

Consideramos que los cinco artículos que componen esta sección especial, dan cuenta de algunos aspectos relevantes respecto al ámbito de la salud mental en la infancia, que tal como dijimos en párrafos anteriores, constituye una etapa vital para el desarrollo humano saludable. 


\section{Referencias}

Abarzúa, M. \& González, M. (2007). Salud mental infanto-juvenil como problemática pública. Revista de Psicología, XVI(2), 79-95.

Brofenbrenner, U. (1979). The Ecology of Human Development. Cambridge, Harvard University Press.

Minoletti, A. \& Zaccaria, A. (2005). Plan Nacional de Salud Mental en Chile: 10 años de experiencia. Revista Panamericana de Salud Pública, 18(4/5), 346-358.

Ministerio de Salud. (2000). Plan Nacional de Salud Mental y Psiquiatría. Santiago: Ministerio de Salud.
World Health Organization. (2001). World Health Report 2001. Mental Health: New Understanding. Ginebra: OMS.

World Health Organization. (2003). Understanding child and adolescent mental disorders: Caring for children and adolescents with mental disorder. Geneva: OMS

World Health Organization (2005) European Ministerial Conference on Mental Health; Facing the Challenges, Building Solutions; Helsinki, WHO.

World Health Organization. (2006). Sistema de salud mental en Chile. Informe de la evaluación del sistema de salud mental en Chile usando World Health Organization - Assessment Instrument for Mental Health Systems (WHO-AIMS). Santiago, Chile: OMS, Ministerio de Salud. 
